\title{
Erratum
}

\section{FTIR Spectroscopic and Theoretical Study of the Photochemistry of Matrix-isolated Coumarin}

\author{
Nihal Kuş, Susana Breda, Igor Reva, Erol Tasal, Cemil Ogretir and Rui Fausto \\ DOI: 10.1111/j.1751-1097.2007.00233.x
}

In the most recent issue of Photochemistry and Photobiology, 2007, 83(5), 1237-1253, an error in Table 2 was published.

The corrected Table 2 is shown:

Table 2. Frequencies $\left(\mathrm{cm}^{-1}\right)$ and intensities $\left(\mathrm{km} \mathrm{mol}^{-1}\right)$ of monomeric coumarin theoretically calculated at the $\mathrm{B} 3 \mathrm{LYP} / 6-311++\mathrm{G}(\mathrm{d}, \mathrm{p})$ and MP2/6-31G $(\mathrm{d}, \mathrm{p})$ levels compared to the observed infrared spectrum of coumarin isolated in an argon matrix at $10 \mathrm{~K}^{*}$.

\begin{tabular}{|c|c|c|c|c|c|c|c|}
\hline \multirow{2}{*}{$\begin{array}{l}\text { Approximate } \\
\text { description } \dagger\end{array}$} & \multirow[b]{2}{*}{ Sym. } & \multicolumn{2}{|c|}{$\begin{array}{c}\text { Calculated } \\
\text { DFT(B3LYP)/ } \\
6-311++ \text { G(d,p) }\end{array}$} & \multicolumn{2}{|c|}{$\begin{array}{c}\text { Calculated } \\
\text { MP2/6-31G(d,p) }\end{array}$} & \multicolumn{2}{|l|}{$\begin{array}{l}\text { Observed spectrum } \\
\qquad \operatorname{Ar}(10 \mathrm{~K})\end{array}$} \\
\hline & & Freq + & Int. & Freq. $\S$ & Int. & Freq. & Int. $\|$ \\
\hline$v(\mathrm{C} 3-\mathrm{H})$ & $\mathrm{A}^{\prime}$ & 3099 & 0.3 & 3100 & 0.5 & - & \\
\hline$v(\mathrm{C} 7-\mathrm{H})$ & $\mathrm{A}^{\prime}$ & 3088 & 3.2 & 3087 & 4.1 & 3096 & 1.0 \\
\hline$v(\mathrm{C} 9-\mathrm{H})$ & $\mathrm{A}^{\prime}$ & 3079 & 11.0 & 3079 & 10.6 & 3085,3079 & 1.5 \\
\hline$v(\mathrm{C} 8-\mathrm{H})$ & $\mathrm{A}^{\prime}$ & 3065 & 7.0 & 3064 & 5.5 & 3059 & 1.0 \\
\hline$v(\mathrm{C} 10-\mathrm{H})$ & $\mathrm{A}^{\prime}$ & 3055 & 5.2 & 3050 & 0.9 & 3053 & 1.1 \\
\hline$v(\mathrm{C} 4-\mathrm{H})$ & $\mathrm{A}^{\prime}$ & 3051 & 4.1 & 3052 & 11.0 & 3043 & 0.7 \\
\hline$v(\mathrm{C}=\mathrm{O})$ & $\mathrm{A}^{\prime}$ & 1772 & 718.0 & 1757 & 422.3 & $1776,1774,1772,1767,1762,1759,1752,1748,1747$ & 456.6 \\
\hline$v(\mathrm{C} 3=\mathrm{C} 4)$ & $\mathrm{A}^{\prime}$ & 1633 & 65.9 & 1628 & 24.8 & $1635,1632,1631,1630$ & 45.0 \\
\hline$v(\mathrm{C} 10-\mathrm{C} 9)$ & $\mathrm{A}^{\prime}$ & 1616 & 60.7 & 1608 & 25.7 & $1617,1613,1612,1610$ & 54.1 \\
\hline$v(\mathrm{C} 8-\mathrm{C} 7)$ & $\mathrm{A}^{\prime}$ & 1568 & 39.3 & 1566 & 6.3 & 1572,1571 & 12.7 \\
\hline$\delta(\mathrm{C}-\mathrm{H}) p h$ & $\mathrm{~A}^{\prime}$ & 1489 & 5.2 & 1484 & 8.4 & 1492 & 3.2 \\
\hline$v(\mathrm{C} 7-\mathrm{C} 6)$ & $\mathrm{A}^{\prime}$ & 1453 & 23.4 & 1445 & 31.6 & $1459,1458,1455$ & 25.8 \\
\hline$\delta(\mathrm{C}-\mathrm{H}) p y$ & $\mathrm{~A}^{\prime}$ & 1400 & 10.1 & 1425 & 11.6 & 1399 & 9.8 \\
\hline$v(\mathrm{C} 5-\mathrm{C} 6) / \delta(\mathrm{C}-\mathrm{H}) p h$ & $\mathrm{~A}^{\prime}$ & 1336 & 2.7 & 1390 & 12.8 & 1329,1327 & 1.9 \\
\hline$\delta(\mathrm{C}-\mathrm{H}) p h / v(\mathrm{C} 5-\mathrm{C} 6)$ & $\mathrm{A}^{\prime}$ & 1273 & 20.7 & 1258 & 27.3 & $1278,1276,1275$ & 13.2 \\
\hline$v(\mathrm{C} 6-\mathrm{O})$ & $\mathrm{A}^{\prime}$ & 1255 & 28.6 & 1242 & 14.0 & $1263,1262,1260,1259$ & 17.6 \\
\hline$v(\mathrm{C} 4-\mathrm{C} 5)$ & $\mathrm{A}^{\prime}$ & 1225 & 13.1 & 1224 & 2.2 & $1233,1232,1228,1226,1225$ & 13.8 \\
\hline$\delta(\mathrm{C}-\mathrm{H}) p y / v(\mathrm{C} 2-\mathrm{C} 3)$ & $\mathrm{A}^{\prime}$ & 1169 & 25.6 & 1174 & 99.5 & $1202,1197,11961194,1181,1178$ & 69.2 \\
\hline$\delta(\mathrm{C}-\mathrm{H}) p h$ & $\mathrm{~A}^{\prime}$ & 1156 & 0.9 & 1151 & 0.9 & 1156,1153 & 1.2 \\
\hline$\delta(\mathrm{C}-\mathrm{H}) \mathrm{ph}$ & $\mathrm{A}^{\prime}$ & 1118 & 20.5 & 1111 & 47.5 & $1134,1132,1129,1128,1123,1119,1118$ & 32.6 \\
\hline$v(\mathrm{C} 2-\mathrm{C} 3) / \delta(\mathrm{C}-\mathrm{H}) p y$ & $\mathrm{~A}^{\prime}$ & 1075 & 99.8 & 1088 & 49.2 & $1106,1102,1098,1097$ & 56.1 \\
\hline$v(\mathrm{C} 9-\mathrm{C} 8)$ & $\mathrm{A}^{\prime}$ & 1030 & 2.2 & 1021 & 1.3 & 1031,1030 & 1.0 \\
\hline$\gamma(\mathrm{C}-\mathrm{H}) p y$ & $\mathrm{~A}^{\prime \prime}$ & 983 & 0.5 & 964 & 0.5 & 984,982 & 0.2 \\
\hline$\gamma(\mathrm{C}-\mathrm{H}) \mathrm{ph}$ & $\mathrm{A}^{\prime \prime}$ & 969 & 0.2 & 917 & 0.3 & - & \\
\hline$\gamma(\mathrm{C}-\mathrm{H}) p h$ & $\mathrm{~A}^{\prime \prime}$ & 948 & 3.2 & 903 & 0.5 & $945,944,942,940$ & 3.4 \\
\hline$v(\mathrm{O}-\mathrm{C} 2) / \delta \operatorname{ring} p h$ & $\mathrm{~A}^{\prime}$ & 922 & 28.8 & 911 & 23.5 & $929,927,926$ & 19.8 \\
\hline$\delta$ ring $p h / v(\mathrm{O}-\mathrm{C} 2)$ & $\mathrm{A}^{\prime}$ & 875 & 43.3 & 949 & 41.5 & 888,886 & 21.0 \\
\hline$\gamma(\mathrm{C}-\mathrm{H}) p h$ & $\mathrm{~A}^{\prime \prime}$ & 863 & 3.1 & 843 & 0.6 & $866,865,864$ & 2.3 \\
\hline$\gamma(\mathrm{C}-\mathrm{H}) p y$ & $\mathrm{~A}^{\prime \prime}$ & 830 & 59.1 & 827 & 61.0 & $832,829,828,827$ & 52.2 \\
\hline$v(\mathrm{C} 10-\mathrm{C} 5)$ & $\mathrm{A}^{\prime}$ & 761 & 2.5 & 785 & $2.6 x$ & $765,763,762,761,759,753$ & 56.2 \\
\hline$\gamma(\mathrm{C}-\mathrm{H}) p h$ & $\mathrm{~A}^{\prime \prime}$ & 761 & 46.8 & 763 & $46.0\}$ & & \\
\hline$\tau \operatorname{ring} p y \Phi$ & $\mathrm{A}^{\prime \prime}$ & 740 & 15.9 & & & & \\
\hline$\delta$ ring $p h$ & $\mathrm{~A}^{\prime}$ & 731 & 2.6 & 754 & 1.7 & 726,725 & 1.1 \\
\hline$\gamma(\mathrm{C}=\mathrm{O})$ & $\mathrm{A}^{\prime \prime}$ & 678 & 0.5 & 669 & 4.2 & 680 & 0.2 \\
\hline$\delta$ ring $p y$ & $\mathrm{~A}^{\prime}$ & 614 & 8.3 & 626 & 9.7 & 610 & 7.3 \\
\hline$\tau \operatorname{ring} p y$ & $\mathrm{~A}^{\prime \prime}$ & 538 & 0.2 & 503 & 0.0 & - & \\
\hline$\delta$ ring $p y$ & $\mathrm{~A}^{\prime}$ & 530 & 6.7 & 542 & 3.9 & 527,526 & 2.0 \\
\hline$\delta(\mathrm{C}=\mathrm{O})$ & $\mathrm{A}^{\prime}$ & 489 & 5.4 & 497 & 3.9 & $490,486(?)$ & 0.6 \\
\hline$\tau$ Butterfly & $\mathrm{A}^{\prime \prime}$ & 454 & 5.2 & 435 & 1.3 & 459,452 & 4.6 \\
\hline$\delta$ ring $p h$ & $\mathrm{~A}^{\prime}$ & 445 & 1.5 & 456 & 0.8 & 446 & 0.3 \\
\hline
\end{tabular}


Table 2. (Continued)

\begin{tabular}{|c|c|c|c|c|c|c|c|}
\hline \multirow{2}{*}{$\begin{array}{l}\text { Approximate } \\
\text { description } \dagger\end{array}$} & \multirow[b]{2}{*}{ Sym. } & \multicolumn{2}{|c|}{$\begin{array}{c}\text { Calculated } \\
\text { DFT(B3LYP)/ } \\
6-311++ \text { G }(\mathrm{d}, \mathrm{p})\end{array}$} & \multicolumn{2}{|c|}{$\begin{array}{c}\text { Calculated } \\
\text { MP2/6-31G(d,p) }\end{array}$} & \multicolumn{2}{|c|}{$\begin{array}{l}\text { Observed spectrum } \\
\quad \operatorname{Ar}(10 \mathrm{~K})\end{array}$} \\
\hline & & Freq $*$ & Int. & Freq. $\S$ & Int. & Freq. & Int.|| \\
\hline$\tau \operatorname{ring} p y$ & $\mathrm{~A}^{\prime \prime}$ & & & 355 & 0.4 & & \\
\hline$\tau$ ring $p h$ & $\mathrm{~A}^{\prime \prime}$ & 369 & 0.1 & 327 & 0.1 & & \\
\hline$\delta$ ring $p y$ & $\mathrm{~A}^{\prime}$ & 304 & 0.8 & 308 & 1.0 & Not investigated & \\
\hline$\tau$ ring $p y$ & $\mathrm{~A}^{\prime \prime}$ & 253 & 0.5 & 243 & 0.3 & - & \\
\hline$\tau$ ring $p h$ & $\mathrm{~A}^{\prime \prime}$ & 154 & 4.6 & 154 & 4.5 & & \\
\hline$\tau$ ring $p h$ & $\mathrm{~A}^{\prime \prime}$ & 93 & 1.4 & 93 & 1.2 & & \\
\hline
\end{tabular}

*See Table S1 for definition of symmetry coordinates and Tables S2 and S3 for PEDs calculated using the DFT and MP2 force constants and geometries, respectively. †Approximate description is known to be an oversimplification of the vibrations description, where their description in terms of a single, most significant symmetry coordinate was attempted. The detailed description is given in the PED form (Tables S2 and S3).

$v$, bond stretching; $\delta$, bending; $\gamma$, rocking; $\tau$, torsion; $p h$, phenyl ring; $p y$, pyrone ring. Wherever two approximate descriptions are given, separated by a slash (/) symbol, the left one corresponds to the approximate description extracted from the PEDs calculated at the DFT(B3LYP)/6$311++\mathrm{G}(\mathrm{d}, \mathrm{p})$ level and the right one to that obtained based on the MP2/6-31G(d,p) calculations. $\$$ Theoretical positions of absorption bands were scaled by a factor 0.964 in the $4000-2500 \mathrm{~cm}^{-1}$ region; 0.982 in the $2500-1000 \mathrm{~cm}^{-1}$ region and 0.989 in the region below $1000 \mathrm{~cm}^{-1}$. $\$$ Theoretical positions of absorption bands were scaled by a factor of 0.938 in the $4000-2500 \mathrm{~cm}^{-1}$ region; 0.956 in the $2500-1000 \mathrm{~cm}^{-1}$ region and 1.014 in the region below $1000 \mathrm{~cm}^{-1}$. $\mid$ Observed intensities ( $\operatorname{Int}_{\text {exp }}$ ) correspond to band integral absorbances $(A)$ normalized by the theoretical intensities

(Int calc $)$ at MP2/6-31G(d,p) level, according to the formula Int $\exp ^{(i)}=A^{(i)} \Sigma \operatorname{Int}_{\text {calc }} / \Sigma A$, where the sums extend to all theoretical bands which have an experimentally observed counterpart. In the low frequency region the torsional vibrations of the pyrone ring were predicted in a substantially different way by the DFT(B3LYP)/6-311 + $+\mathrm{G}(\mathrm{d}, \mathrm{p})$ and MP2/6-31G(d,p) methods. 
Copyright of Photochemistry \& Photobiology is the property of Blackwell Publishing Limited and its content may not be copied or emailed to multiple sites or posted to a listserv without the copyright holder's express written permission. However, users may print, download, or email articles for individual use. 